\title{
Theoretical and Experimental Investigation of Superplastic Rolling Process
}

\author{
Hani Aziz Ameen and Rahman Ali Hussain \\ Technical College - Baghdad/ Iraq. \\ Dies and Tools Engineering Dept. \\ E-mail: haniazizameen@yahoo.com
}

\begin{abstract}
Rolling play an increasingly significant role in forming process, very few studies have been devoted to the case of rolling superplastic alloy. This paper presents an investigation of symmetrical and unsymmetrical rolling process, which is of fundamental interest for forming processes. The prediction of the strain rate in the unsymmetrical rolling process is thus a challenging task. This paper has a novel discussion of symmetrical and unsymmetrical rolling of superplastic (lead-tin) eutectic alloy and with different range of rolling speed ratios and percentage reduction in thickness. It was found that the strain rate calculated experimentally was always greater than that calculated from theoretical considerations. Curling of the specimen as it leaves the rolls in the unsymmetrical rolling depends on the percentage reduction in thickness and on roll speed ratio.
\end{abstract}

Keywords: superplastic material, symmetrical rolling, unsymmetrical rolling, (Lead-Tin) eutectic alloy.

\section{INTRODUCTION}

Superplastic materials are a unique class of polycrystalline solids that have the ability to undergo very large, uniform tensile elongations prior to failure. Elongations in excess of $200 \%$ usually indicate superplasticity. Rolling is an important forming process in diverse fabrications, especially with the emergence of industrial development due to the need of ferrous and nonferrous sections of different shapes for machines, apparatus and industrial structure [1]. The rolling process divided into two types; the first type is symmetrical rolling, this is a common process. It consists of passing the work piece between two rolls of equal diameters, rotational speeds and surface fineness. The rolled metal exists at a lower thickness with maintenance of its straightness. The another type is non-symmetrical rolling in which the workpieces are rolled between two rolls of different radii, rotational speeds or surface fineness. The workpiece exists from the roll gap curved in a direction dependent on the difference in above variables. Detailed studies are conducted on rolling processes due to its importance and need in all industries. These elaborate on the required loads and moments for forming as well as the theoretical/experimental factors influencing it, resulting stresses and strains due to forming by rolling. Such studies were conducted more on symmetrical rolling than on non-symmetrical rolling of strain rate sensitive materials. Orowan, 1943, [2] established and improved the theoretical assumptions for the rolling process. Bland and Ford,1950, [3] simplified the theoretical solutions established by Orwan by adding further assumption by addition of a front and rear tension force for the work during rolling. Alto,1976, [4] was an early researcher at implementation of strain rate sensitive materials in the symmetrical rolling process. He studied the behavior of lead-tin alloy and found the equation of the case that makes the alloy behave like a plastic. Dewhurs et al., 1973,[5] and Johnson and Needham,1982, [6] conducted experiments on non- symmetrical rolling of lead slabs. Chelyshev and Luzhnyl,1988, [7] studied the plastic curvature of a slab upon its rolling non- symmetrically with respect to difference of rolls' diameters. They established mathematical relationships to calculate the curvature radius of the rolled workpiece. Nikolaev,1978, [8] conducted experiments on aluminum slabs to study the effect of introducing the workpieces to rolling process so as to make an angle with the horizon. Similarly, Nikolaev et al.,1988,[9] conducted experiments on slabs of 2layers aluminum separated by steel layer. They 
established mathematical relationship that define the distribution of stresses and moments between the rolls. Researches indicate existence of, at least, 126 metals or alloys with superplasticity property [10], these materials may be categorized on the basis of the temperature at which the metal acquires the superplasticity property in two categories, alloy with $(100-300)^{\circ} \mathrm{C}$ low melting points, e.g. alloys of lead-tin, lead-cadmium, etc. These acquire the superplasticity property at nearly room temperature, the other alloys with medium (about $500^{\circ} \mathrm{C}$ ) melting points, e.g. zinc- 140 aluminum. Chung and Cheng, 2001,[11] established integrated numerical procedures to effectively simulate the superplastic forming process for complex shaped components. They further carried out stability analysis to investigate necking in superplastic material characterized by the sinh-law constitutive equation. They plotted a necking map, by observing the effect of load and strain rate sensitivity parameter on necking, under uniaxial loading conditions.

In this study, designing and execution of a symmetrical The speed, $\omega_{2}$ enters the gearbox(3) where goes and non-symmetrical rolling machine from which low another reduction by $1 / 60$.

strain rate consistent for forming superplastic materials may be obtained, conducting experiments to find strain rate in different cross section during rolling and its comparison with theoretical solutions. Lead-Tin eutectic alloy was chosen for the experiments after imparting to div it superplastic property to represent the materials sensitive to strain rate.

\section{Design and Execution of the Rolling Machine} Practical experiments require the gain of low strain $V_{4}=r_{1} \omega_{4}=66 \times 0.243=16.038 \mathrm{~mm} / \mathrm{sec}$, where $r_{1}$ is rates during rolling processes suitable for superplastic the radius of the first pulley $=66 \mathrm{~mm}$.

materials. Therefore, the rolling machine should be Speed is transmitted by the transmission belt(5) whose capable of acquiring slow rotational speeds. Thus a length can be calculated as follow:

machine was designed and executed in which the following conditions are available.

1- Generation of low strain rates of around $\left(10^{-2}-10^{-4}\right)$ where:

$$
\begin{gathered}
L=\frac{1}{2} \pi\left(D_{1}+D_{2}\right)+\alpha\left(D_{2}-D_{1}\right)+\sqrt{4 C^{2}-\left(D_{2}-D_{1}\right)^{2}} \\
=1300 \mathrm{~mm}
\end{gathered}
$$

$D_{1}, D_{2}=$ diameter of the first and second pulley $=75$ /sec.

and $135 \mathrm{~mm}$ and

2- Possibility of change of speed between the rolls to
obtain non-symmetrical rolling as well as symmetrical speeds so as to attain conditions similar to the conditions of the experiments of the researcher Alto, 1976, [4] for comparison.

3- Generation of enough torque to conduct the rolling process.

$$
\alpha=\text { inclination angle }=\sin ^{-1} \frac{D_{2}-D_{1}}{2 C}=0.062 \mathrm{rad}
$$

Since the linear speed of the first pulley $=$ the linear speed of the second pulley

$$
V_{4}=r_{2} \omega_{5} \Rightarrow \omega_{5}=\frac{16.038}{40}=0.4 \mathrm{rad} / \mathrm{sec}
$$


Speed $\omega_{5}$ enters into the second gearbox(8), where it is cylinder (10), thus working the output speed for that roll completely reduced by $1 / 435$. Thus the output speed at equal to $0.0088 \mathrm{rpm}$. Table1 illustrates the the second gearbox $(8)$ is

$$
\omega_{6}=0.4 \times \frac{1}{435}=0.00092 \mathrm{rad} / \mathrm{sec}
$$

specifications of the alloy steel gears. It was possible to obtain different speed reduction rates between the two

The speed $\omega_{6}$ is directly transported by a universal shaft and front flange and a back flange to the bottom rolling

Table1:The Specification of Gears of Speed Reduction Between the Rolls

\begin{tabular}{|c|c|c|c|c|c|}
\hline Gear No. & No. of Teeth & Module (mm) & Depth(mm) & Width(mm) & Dia. Pitch(mm) \\
\hline 1 & 35 & 4 & 8 & 40 & 140 \\
\hline 2 & 38 & 4 & 8 & 40 & 152 \\
\hline 3 & 39 & 4 & 8 & 40 & 156 \\
\hline 4 & 40 & 4 & 8 & 40 & 160 \\
\hline 5 & 40 & 4 & 8 & 40 & 160 \\
\hline 6 & 43 & 4 & 8 & 40 & 172 \\
\hline 7 & 43 & 4 & 8 & 40 & 172 \\
\hline 8 & 45 & 4 & 8 & 40 & 180 \\
\hline
\end{tabular}

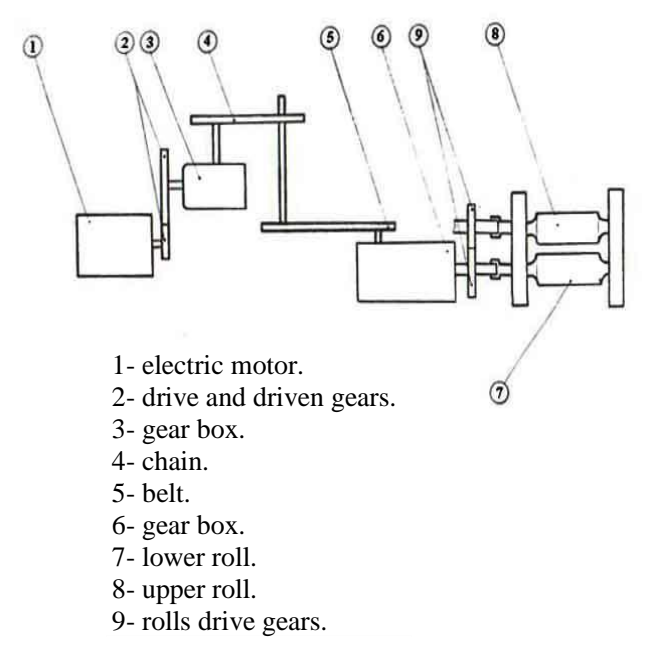

Fig 1: The sketch of the proposal machine design.

Experimental and Theoretical Considerations: A lead - tin eutectic alloy was used for rolling experiments, this alloy acquire superplastic property upon formation with specific reduction in ambient temperature, therefore, the specimens should be cold formed to ensure that the alloys acquires superplastic property.

Method of Preparing the Alloy: The precise weight structure for the constituents of the alloy is (lead $-61.9 \%$ tin). The appropriate weight of both pure metals are measured. For weight accuracy a

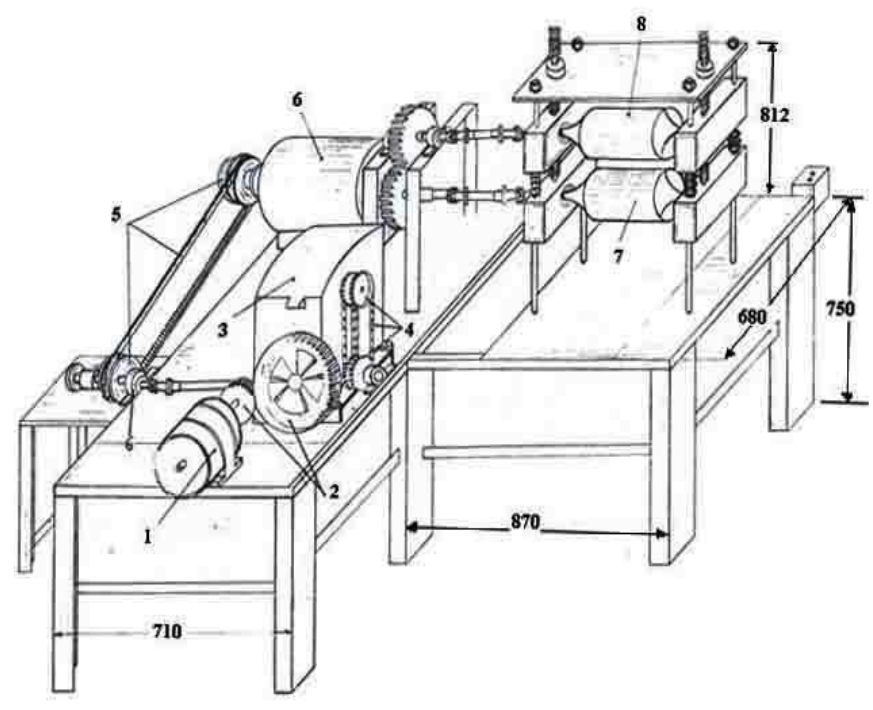

Fig 2: Shows the rolling machine

quantity of the metals are cut in the form of chip for balance. A 0.1 gram sensitivity scale was used to prepare the weights. After preparing the appropriate weights, the metals are melts in a pre-heater ceramic crucible by use of simple gas torch. The materials are mixed with stirring for homogeneity. The melt is poured in two types of pre-heater metal casting molds in order to obtain alloys of two dimensions as follows:

1- The melt is poured in a cubic metal mold open from the top (as in Figure 3 ) to produce a 
$(60 \times 60 \times 85) \mathrm{mm}$ cast after leaving it for a sufficient time to cool. It is used to prepare specimens for rolling experiments.

2- The melt is poured in a cylindrical metal mold (as in Figure 4) to produce a $(\varnothing 27 \times 120) \mathrm{mm}$ cast. It is used to prepare specimen for mechanical testing.
Preparations of the specimens: The aim is to obtain a micro grain size in the range of (1-10) microns in order to impart to this cast superplastic property. This requires cold forming by mechanical pressing, rolling and extrusion to prepare the different specimens as follows:
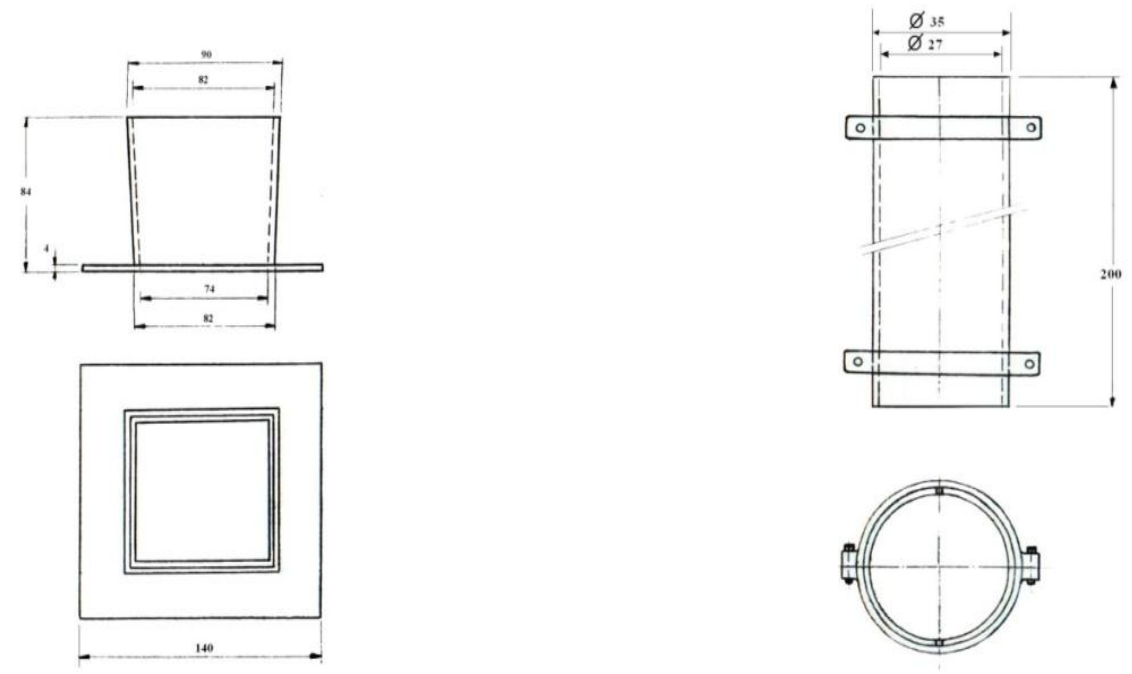

Fig 3: Shows the cylindrical mold

Rolling specimens: They are specimens used to conduct rolling process. The first alloys were used to prepare these specimens. After taking the cast out of the mold, the faces were straightened by milling operation, and then pressed by a $200 \mathrm{kN}$ Avery-Denison hydraulic press using a $1000 \mathrm{kN}$ load to reduce the casts thickness to $10 \mathrm{~mm}$. This $85.6 \%$ reduction was obtained. The following step was determining four specimens for shearing to $(10 \times 95 \times 50) \mathrm{mm}$ dimensions by a guillotine shearing machine. This was followed by another reduction by rolling to obtain the dimensions $(5.5 \times 100 \times 50) \mathrm{mm}$.

Specimen of Mechanical Testing: They are used to prepare the specimens for conducting experiments of mechanical testing. After extracting the cast rod of $(\varnothing 27 \times 120) \mathrm{mm}, 20 \mathrm{~mm}$ is cut from both terminals to insure the homogeneity of the micro-grain composition along the remaining length which cleans its surface by this operation. Then, it is cut into two equal specimens; each one is used to perform forming operation by extrusion to obtain a $12 \mathrm{~mm}$ radius bar, this is cut into $24 \mathrm{~mm}$ specimens by using a disc saw with five teeth.
(Figure 5) illustrates the used extrusion mold for this purpose.

\section{Practical Experiments \\ Finding the Equation of the Mechanical Properties of an Eutectic Lead-Tin Alloy: The} description of the alloy during the forming process requires finding the equation of the mechanical properties.

$\sigma=K \dot{\varepsilon}^{m}$

Therefore, the compression experiment was performed on Instron device for testing the tension and compression for $12 \mathrm{~mm}$ radius, $24 \mathrm{~mm}$ length specimens. The speed of piston movement during pressing the specimen changes from $0.5 \mathrm{~mm} / \mathrm{min}$ to $20 \mathrm{~mm} / \mathrm{min}$ to insure obtaining strain rates for the specimen in the range $\left(10^{-2}-10^{-4}\right) / \mathrm{sec}$, from the force- elongation curve obtained from the experiment, it can be drawn the strain rate-stress curve, hence the value of $m=0.475$ and $K=29339 \mathrm{~N}$. $\mathrm{sec} / \mathrm{mm}^{2}$ (Figure 6 illustrates this relationship). 
Am. J. Sci. Ind. Res., 2013, 4(1): 59-74

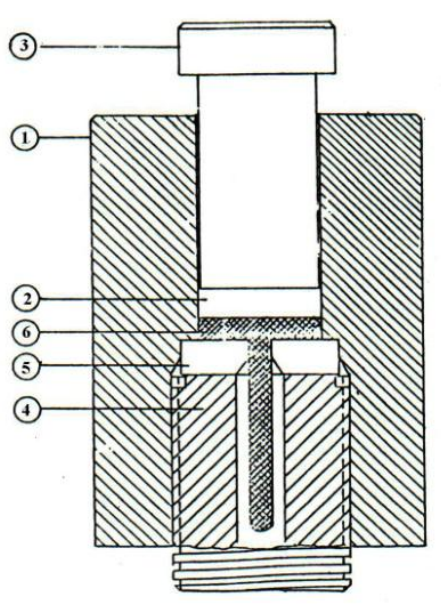

Fig 5: illustrates the used extrusion mold

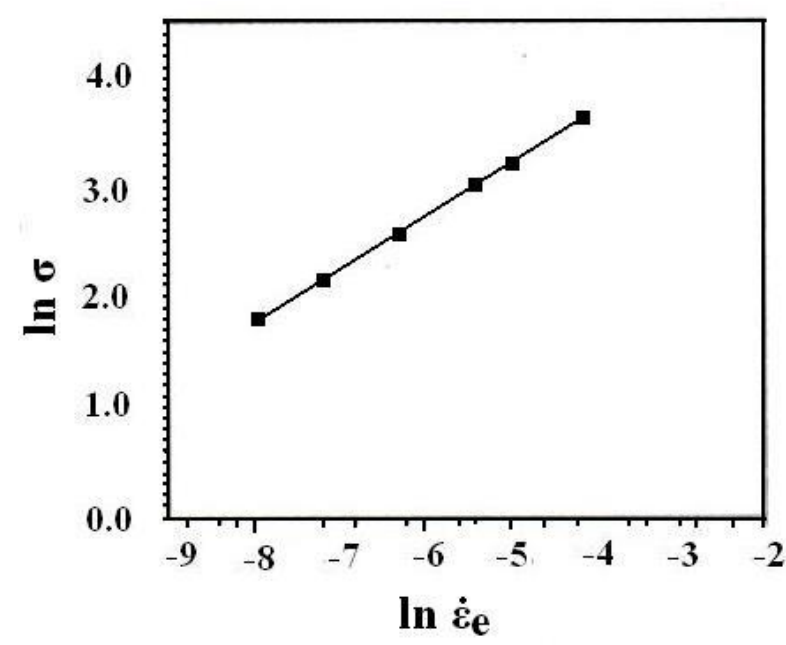

Fig 6: illustrates the relationship between log (stress and equivalent strain rate
Obtaining the State of Plane Strain: In order to obtain the dimensions of the specimens for rolling experiments, it is necessary to perform experiments to obtain the state of rolling by plane strain. Specimens of different dimensions were used. The method of photoplasticity to measure the flow of metal and, therefore, its strains was implemented. A grid was printed on the thickness of each specimen by using grid printing plates. The specimen is supported between the supported plates, fixed in the holder and placed on the base of the compression device. Figure 7 shows the method of fixing the specimen.

The grid printing plate was fixed on the specimen's thickness after ensuring the parallels of the grid's edge with that of the specimen, and then the plate

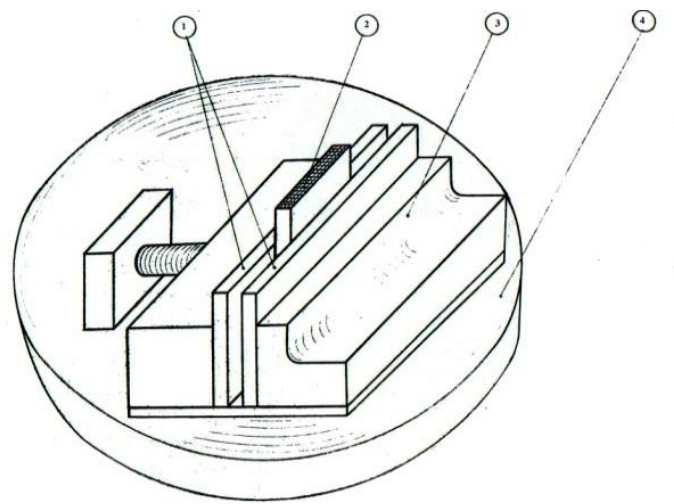

Fig 7: Shows the method of fixing the specimen is pressed from the top by applying a gradual load of up to $5 \mathrm{kN}$. After the end of this operation, the pressing load lifted and the specimen extracted on its thickness is printed the trace of grid of squares. After microscopic measurement the original dimension of the squares grid in the $x$ and $y$ direction, a symmetrical rolling process is performed on the specimens, the deformations are tested to find the strains that occurred to the grid. Tables 2, 3 and 4 illustrate the dimension of the specimens PS1, PS2 and PS3 used in the experimental of plane strain. Figure 8 illustrates the printing of the grid on the specimen's thickness.

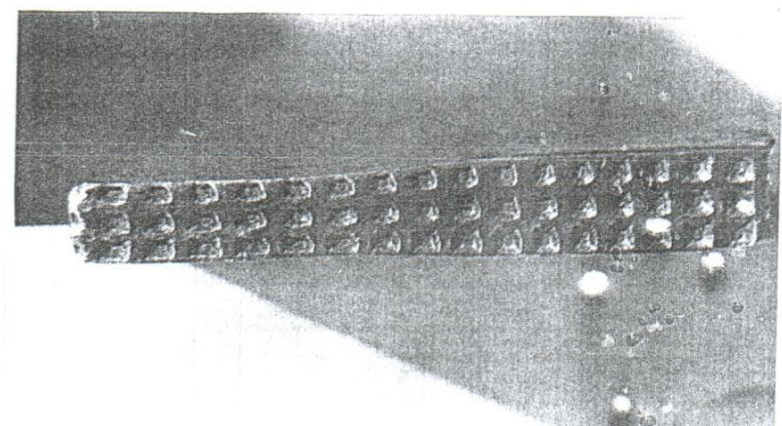

Fig 8: Shows the printed specimen 
Table2:Illustrates the Dimensions of Specimen (PS1) Before and After Symmetrical Rolling in Plane Strain Experiment.

\begin{tabular}{|c|c|c|c|c|}
\hline Square & $\begin{array}{c}\mathbf{d x}(\mathbf{m m}) \\
\text { before } \\
\text { rolling }\end{array}$ & $\begin{array}{c}\mathbf{d x}(\mathbf{m m}) \\
\text { after rolling }\end{array}$ & $\begin{array}{c}\text { dy }(\mathbf{m m}) \\
\text { before } \\
\text { rolling }\end{array}$ & $\begin{array}{c}\mathbf{d y} \\
\mathbf{( m m}) \\
\text { after } \\
\text { rolling }\end{array}$ \\
\hline 1 & 0.41 & 0.501 & 0.51 & 0.36 \\
\hline 2 & 0.48 & 0.62 & 0.43 & 0.295 \\
\hline 3 & 0.50 & 0.747 & 0.41 & 0.25 \\
\hline 4 & 0.57 & 0.81 & 0.50 & 0.33 \\
\hline 5 & 0.55 & 0.744 & 0.58 & 0.416 \\
\hline
\end{tabular}

The original thickness of the specimen $=5.5 \mathrm{~mm}$, The final thickness of the specimen $=4.94 \mathrm{~mm}$, Reduction ratio $=0.2 \%$, Rolling angle $=50^{\circ}$

Table3:Illustrates the Dimensions of Specimen (PS2) Before and After Symmetrical Rolling in Plane Strain Experiment.

\begin{tabular}{|c|c|c|c|c|c|}
\hline Square & $\begin{array}{c}\mathrm{dx} \\
(\mathrm{mm}) \\
\text { before } \\
\text { rolling }\end{array}$ & $\begin{array}{c}\mathrm{dx}(\mathrm{mm}) \\
\text { after } \\
\text { rolling }\end{array}$ & $\begin{array}{c}\text { dy } \\
(\mathrm{mm}) \\
\text { before } \\
\text { rolling }\end{array}$ & $\begin{array}{c}\text { dy } \\
(\mathrm{mm}) \\
\text { after } \\
\text { rolling }\end{array}$ & $\begin{array}{l}\text { Square } \\
\text { row }\end{array}$ \\
\hline 1 & 0.81 & 0.99 & 0.64 & 0.50 & \multirow{5}{*}{ Upper } \\
\hline 2 & 0.41 & 0.50 & 0.634 & 0.498 & \\
\hline 3 & 0.45 & 0.52 & 0.60 & 0.50 & \\
\hline 4 & 0.575 & 0.64 & 0.68 & 0.60 & \\
\hline 5 & 0.44 & 0.48 & 0.64 & 0.58 & \\
\hline 1 & 0.446 & 0.60 & 0.60 & 0.40 & \multirow{5}{*}{ Medium } \\
\hline 2 & 0.56 & 0.69 & 0.60 & 0.44 & \\
\hline 3 & 0.81 & 0.99 & 0.67 & 0.50 & \\
\hline 4 & 0.48 & 0.54 & 0.605 & 0.506 & \\
\hline 5 & 0.405 & 0.52 & 0.60 & 0.45 & \\
\hline
\end{tabular}

The original thickness of the specimen $=7 \mathrm{~mm}$, The final thickness of the specimen $=6.6 \mathrm{~mm}$, Reduction ratio $=5.7 \%$, Rolling angle $=4.65^{\circ}$

Table4:Illustrates the Dimensions of Specimen (PS3) Before And After Symmetrical Rolling in Plane Strain Experiment.

\begin{tabular}{|c|c|c|c|c|c|}
\hline Square & $\begin{array}{c}\text { dx } \\
(\mathrm{mm}) \\
\text { before } \\
\text { rolling }\end{array}$ & $\begin{array}{c}\mathrm{dx}(\mathrm{mm}) \\
\text { after } \\
\text { rolling }\end{array}$ & $\begin{array}{c}\text { dy } \\
(\mathrm{mm}) \\
\text { before } \\
\text { rolling }\end{array}$ & $\begin{array}{c}\text { dy } \\
(\mathbf{m m}) \\
\text { after } \\
\text { rolling }\end{array}$ & $\begin{array}{c}\text { Square } \\
\text { row }\end{array}$ \\
\hline 1 & 0.65 & 0.75 & 0.72 & 0.63 & \multirow{5}{*}{ Medium } \\
\hline 2 & 0.63 & 0.75 & 0.72 & 0.6 & \\
\hline 3 & 0.55 & 0.76 & 0.75 & 0.54 & \\
\hline 4 & 0.41 & 0.46 & 0.72 & 0.64 & \\
\hline 5 & 0.6 & 0.61 & 0.73 & 0.72 & \\
\hline 1 & 0.6 & 0.75 & 0.60 & 0.48 & \multirow{5}{*}{ Lower } \\
\hline 2 & 0.63 & 0.78 & 0.60 & 0.48 & \\
\hline 3 & 0.50 & 0.59 & 0.62 & 0.53 & \\
\hline 4 & 0.42 & 0.52 & 0.59 & 0.48 & \\
\hline 5 & 0.40 & 0.49 & 0.59 & 0.58 & \\
\hline
\end{tabular}

The original thickness of the specimen $=5.5 \mathrm{~mm}$, The final thickness of the specimen $=5.1 \mathrm{~mm}$, Reduction ratio $=9.1 \%$, Rolling angle $=4.66^{\circ}$

Experiments of Symmetrical Rolling: The machine is ready for symmetrical rolling after fixing a pair of gears which symmetrically transmits motion to both rolls. A grid print was made on the thickness of each specimen. The dimensions of the original grids were also measured. Tables 5 and 6 illustrate the dimensions of the grid before and after symmetrical rolling on specimens S1 and S2.

Table 5: Illustrates the Dimensions of Specimen (S1) Before and After Symmetrical Rolling in Plane Strain Experiment.

\begin{tabular}{|c|c|c|c|c|c|}
\hline Square & $\begin{array}{c}\text { dx } \\
(\mathrm{mm}) \\
\text { before } \\
\text { rolling }\end{array}$ & $\begin{array}{c}\mathbf{d x}(\mathbf{m m}) \\
\text { after } \\
\text { rolling }\end{array}$ & $\begin{array}{c}\text { dy } \\
(\mathrm{mm}) \\
\text { before } \\
\text { rolling }\end{array}$ & $\begin{array}{c}\text { dy } \\
(\mathrm{mm}) \\
\text { after } \\
\text { rolling }\end{array}$ & $\begin{array}{c}\text { Squa } \\
\text { re } \\
\text { row }\end{array}$ \\
\hline 1 & 0.48 & 0.55 & 0.78 & 0.66 & \multirow{5}{*}{$\begin{array}{c}\text { Medic } \\
\text { m }\end{array}$} \\
\hline 2 & 0.52 & 0.60 & 0.76 & 0.66 & \\
\hline 3 & 0.61 & 0.66 & 0.72 & 0.63 & \\
\hline 4 & 0.60 & 0.65 & 0.71 & 0.64 & \\
\hline 5 & 0.53 & 0.56 & 0.76 & 0.73 & \\
\hline 1 & 0.45 & 0.46 & 0.58 & 0.49 & \multirow{5}{*}{ Lowe } \\
\hline 2 & 0.44 & 0.51 & 0.58 & 0.50 & \\
\hline 3 & 0.58 & 0.65 & 0.585 & 0.52 & \\
\hline 4 & 0.57 & 0.62 & 0.6 & 0.55 & \\
\hline 5 & 0.51 & 0.52 & 0.62 & 0.59 & \\
\hline
\end{tabular}

The original thickness of the specimen $=5.5 \mathrm{~mm}$, The final thickness of the specimen $=5 \mathrm{~mm}$, Reduction ratio $=9.1 \%$, time $=155 \mathrm{sec}$.

Table 6: Illustrates the Dimensions of Specimen (S2) Before and After Symmetrical Rolling in Plane Strain Experiment.

\begin{tabular}{|c|c|c|c|c|c|}
\hline Square & $\begin{array}{c}\mathrm{dx} \\
(\mathrm{mm}) \\
\text { before } \\
\text { rolling }\end{array}$ & $\begin{array}{c}\mathrm{dx}(\mathrm{mm}) \\
\text { after } \\
\text { rolling }\end{array}$ & $\begin{array}{c}\text { dy } \\
\text { (mm) } \\
\text { before } \\
\text { rolling }\end{array}$ & $\begin{array}{c}\text { dy } \\
(\mathrm{mm}) \\
\text { after } \\
\text { rolling }\end{array}$ & $\begin{array}{c}\text { Squa } \\
\text { re } \\
\text { row }\end{array}$ \\
\hline 1 & 0.44 & 0.66 & 0.72 & 0.53 & \multirow{6}{*}{$\begin{array}{l}\text { Mediu } \\
\text { m }\end{array}$} \\
\hline 2 & 0.46 & 0.68 & 0.74 & 0.54 & \\
\hline 3 & 0.52 & 0.73 & 0.74 & 0.59 & \\
\hline 4 & 0.52 & 0.69 & 0.67 & 0.62 & \\
\hline 5 & 0.56 & 0.65 & 0.77 & 0.68 & \\
\hline 6 & 0.68 & 0.67 & 0.62 & 0.61 & \\
\hline 1 & 0.47 & 0.65 & 0.63 & 0.42 & \multirow{6}{*}{ Lower } \\
\hline 2 & 0.35 & 0.51 & 0.62 & 0.45 & \\
\hline 3 & 0.43 & 0.61 & 0.60 & 0.48 & \\
\hline 4 & 0.51 & 0.63 & 0.64 & 0.53 & \\
\hline 5 & 0.60 & 0.67 & 0.62 & 0.55 & \\
\hline 6 & 0.69 & 0.71 & 0.61 & 0.60 & \\
\hline
\end{tabular}

The original thickness of the specimen $=5.62 \mathrm{~mm}$, The final thickness of the specimen $=4.42 \mathrm{~mm}$, Reduction ratio $=21.3 \%$, time $=200 \mathrm{sec}$.

Experiments of non- Symmetrical Rolling: Experiments of non- symmetrical rolling for specimens were performed by changing the inter- 
rolls velocity by using a pair of gears which transmits motion by a specified reduction to the rolls. This reduction depends on the ratio between the number of driven gear's teeth (Z2) and that of the driver gear $(Z 1)$. The choice for speed ratio of the rolls were $(1.184,1.13,1.075)$. These depend on method of engagement of the gears between the rolls in order to be similar to those implemented in former researches. The experiments of nonsymmetrical rolling were conducted after microscopic measurement of the squares of the original grid. Tables 7 to 12 illustrate the dimensions of the grid before and after rolling for the deformed points of specimens S3 to S8.

Table7:Illustrates the Dimensions of Specimen (S3) Before and After Non-Symmetrical Rolling for Gear Ratio Between Rollers (1.075).

\begin{tabular}{|c|c|c|c|c|c|}
\hline Square & $\begin{array}{c}\mathrm{dx} \\
(\mathrm{mm}) \\
\text { before } \\
\text { rolling }\end{array}$ & $\begin{array}{c}\mathrm{dx}(\mathrm{mm}) \\
\text { after } \\
\text { rolling }\end{array}$ & $\begin{array}{c}\text { dy } \\
(\mathrm{mm}) \\
\text { before } \\
\text { rolling }\end{array}$ & $\begin{array}{c}\text { dy } \\
(\mathbf{m m}) \\
\text { after } \\
\text { rolling }\end{array}$ & $\begin{array}{c}\text { Square } \\
\text { row }\end{array}$ \\
\hline 1 & 0.59 & 0.70 & 0.50 & 0.41 & \multirow{6}{*}{ upper } \\
\hline 2 & 0.55 & 0.655 & 0.61 & 0.51 & \\
\hline 3 & 0.46 & 0.55 & 0.47 & 0.40 & \\
\hline 4 & 0.51 & 0.60 & 0.62 & 0.525 & \\
\hline 5 & 0.57 & 0.65 & 0.63 & 0.59 & \\
\hline 6 & 0.49 & 0.50 & 0.64 & 0.63 & \\
\hline 1 & 0.55 & 0.64 & 0.63 & 0.5 & \multirow{6}{*}{ Medium } \\
\hline 2 & 0.41 & 0.49 & 0.68 & 0.57 & \\
\hline 3 & 0.55 & 0.64 & 0.65 & 0.56 & \\
\hline 4 & 0.42 & 0.46 & 0.66 & 0.60 & \\
\hline 5 & 0.61 & 0.64 & 0.68 & 0.66 & \\
\hline 6 & 0.47 & 0.48 & 0.71 & 0.72 & \\
\hline 1 & 0.54 & 0.74 & 0.70 & 0.51 & \multirow{6}{*}{ Lower } \\
\hline 2 & 0.43 & 0.67 & 0.73 & 0.47 & \\
\hline 3 & 0.50 & 0.63 & 0.55 & 0.435 & \\
\hline 4 & 0.50 & 0.60 & 0.76 & 0.69 & \\
\hline 5 & 0.57 & 0.64 & 0.78 & 0.69 & \\
\hline 6 & 0.53 & 0.54 & 0.77 & 0.75 & \\
\hline
\end{tabular}

The original thickness of the specimen $=5.44 \mathrm{~mm}$, The final thickness of the specimen $=4.88 \mathrm{~mm}$, Reduction ratio $=10.3 \%$, time $=180 \mathrm{sec}$.

Table 8: Illustrates the Dimensions of Specimen (S4) Before and After Non-Symmetrical Rolling for Gear Ratio Between Rollers (1.075).

\begin{tabular}{|c|c|c|c|c|c|}
\hline Square & $\begin{array}{c}\mathbf{d x} \\
(\mathrm{mm}) \\
\text { before } \\
\text { rolling }\end{array}$ & $\begin{array}{c}\mathrm{dx}(\mathrm{mm}) \\
\text { after } \\
\text { rolling }\end{array}$ & $\begin{array}{c}\text { dy } \\
\text { (mm) } \\
\text { before } \\
\text { rolling }\end{array}$ & $\begin{array}{c}\text { dy } \\
(\mathbf{m m}) \\
\text { after } \\
\text { rolling }\end{array}$ & $\begin{array}{c}\text { Square } \\
\text { row }\end{array}$ \\
\hline 1 & 0.46 & 0.58 & 0.57 & 0.35 & \multirow{6}{*}{ upper } \\
\hline 2 & 0.42 & 0.49 & 0.59 & 0.44 & \\
\hline 3 & 0.35 & 0.43 & 0.59 & 0.41 & \\
\hline 4 & 0.46 & 0.56 & 0.59 & 0.44 & \\
\hline 5 & 0.62 & 0.70 & 0.58 & 0.47 & \\
\hline 6 & 0.28 & 0.29 & 0.61 & 0.56 & \\
\hline 1 & 0.47 & 0.52 & 0.77 & 0.62 & \multirow{2}{*}{ Medium } \\
\hline 2 & 0.46 & 0.55 & 0.76 & 0.62 & \\
\hline
\end{tabular}

\begin{tabular}{|c|c|c|c|c|c|}
\hline 3 & 0.47 & 0.58 & 0.72 & 0.41 & \\
\hline 4 & 0.535 & 0.62 & 0.76 & 0.67 & \\
\hline 5 & 0.63 & 0.69 & 0.77 & 0.70 & \\
\hline 6 & 0.45 & 0.44 & 0.76 & 0.75 & \\
\hline 1 & 0.50 & 0.59 & 0.52 & 0.50 & \multirow{6}{*}{ Lower } \\
\hline 2 & 0.40 & 0.47 & 0.65 & 0.46 & \\
\hline 3 & 0.435 & 0.50 & 0.64 & 0.38 & \\
\hline 4 & 0.41 & 0.48 & 0.65 & 0.53 & \\
\hline 5 & 0.54 & 0.60 & 0.54 & 0.47 & \\
\hline 6 & 0.47 & 0.50 & 0.74 & 0.70 & \\
\hline
\end{tabular}

The original thickness of the specimen $=5.45 \mathrm{~mm}$, The final thickness of the specimen $=4.56 \mathrm{~mm}$, Reduction ratio $=16.3 \%$, time $=190 \mathrm{sec}$.

Table 9:Illustrates the Dimensions of Specimen (S5) Before and After Non-Symmetrical Rolling for Gear Ratio Between Rollers (1.13).

\begin{tabular}{|c|c|c|c|c|c|}
\hline Square & $\begin{array}{c}\mathrm{dx} \\
(\mathrm{mm}) \\
\text { before } \\
\text { rolling }\end{array}$ & $\begin{array}{c}\mathrm{dx}(\mathrm{mm}) \\
\text { after rolling }\end{array}$ & $\begin{array}{c}\text { dy } \\
\text { (mm) } \\
\text { before } \\
\text { rolling }\end{array}$ & $\begin{array}{c}\text { dy } \\
(\mathrm{mm}) \\
\text { after } \\
\text { rolling }\end{array}$ & $\begin{array}{l}\text { Square } \\
\text { row }\end{array}$ \\
\hline 1 & 0.42 & 0.45 & 0.59 & 0.38 & \multirow{5}{*}{ upper } \\
\hline 2 & 0.49 & 0.54 & 0.57 & 0.37 & \\
\hline 3 & 0.62 & 0.65 & 0.56 & 0.39 & \\
\hline 4 & 0.63 & 0.67 & 0.57 & 0.44 & \\
\hline 5 & 0.43 & 0.47 & 0.54 & 0.46 & \\
\hline 1 & 0.39 & 0.43 & 0.59 & 0.47 & \multirow{5}{*}{ Medium } \\
\hline 2 & 0.48 & 0.585 & 0.61 & 0.46 & \\
\hline 3 & 0.52 & 0.66 & 0.53 & 0.47 & \\
\hline 4 & 0.54 & 0.59 & 0.65 & 0.57 & \\
\hline 5 & 0.37 & 0.38 & 0.61 & 0.58 & \\
\hline 1 & 0.47 & 0.58 & 0.56 & 0.45 & \multirow{5}{*}{ Lower } \\
\hline 2 & 0.40 & 0.48 & 0.57 & 0.45 & \\
\hline 3 & 0.39 & 0.47 & 0.58 & 0.48 & \\
\hline 4 & 0.64 & 0.66 & 0.56 & 0.49 & \\
\hline 5 & 0.56 & 0.60 & 0.48 & 0.45 & \\
\hline
\end{tabular}

The original thickness of the specimen $=5.525 \mathrm{~mm}$, The final thickness of the specimen $=5 \mathrm{~mm}$, Reduction ratio $=9.5 \%$, time $=170 \mathrm{sec}$.

Table 10: Illustrates the Dimensions of Specimen (S6) Before and After Non-Symmetrical Rolling for Gear Ratio Between Rollers (1.13).

\begin{tabular}{|c|c|c|c|c|c|}
\hline Square & $\begin{array}{c}\mathrm{dx} \\
(\mathrm{mm}) \\
\text { before } \\
\text { rolling }\end{array}$ & $\begin{array}{c}\mathrm{dx}(\mathrm{mm}) \\
\text { after rolling }\end{array}$ & $\begin{array}{c}\text { dy } \\
(\mathrm{mm}) \\
\text { before } \\
\text { rolling }\end{array}$ & $\begin{array}{c}\text { dy } \\
(\mathrm{mm}) \\
\text { after } \\
\text { rolling }\end{array}$ & $\begin{array}{l}\text { Square } \\
\text { row }\end{array}$ \\
\hline 1 & 0.59 & 0.80 & 0.64 & 0.45 & \multirow{5}{*}{ upper } \\
\hline 2 & 0.48 & 0.66 & 0.63 & 0.46 & \\
\hline 3 & 0.49 & 0.63 & 0.61 & 0.47 & \\
\hline 4 & 0.50 & 0.61 & 0.53 & 0.46 & \\
\hline 5 & 0.54 & 0.58 & 0.61 & 0.55 & \\
\hline 1 & 0.58 & 0.80 & 0.65 & 0.46 & \multirow{5}{*}{ Medium } \\
\hline 2 & 0.44 & 0.61 & 0.62 & 0.45 & \\
\hline 3 & 0.47 & 0.61 & 0.64 & 0.49 & \\
\hline 4 & 0.46 & 0.57 & 0.65 & 0.53 & \\
\hline 5 & 0.46 & 0.52 & 0.68 & 0.60 & \\
\hline 1 & 0.61 & 0.76 & 0.70 & 0.45 & \multirow{5}{*}{ Lower } \\
\hline 2 & 0.50 & 0.59 & 0.78 & 0.46 & \\
\hline 3 & 0.47 & 0.62 & 0.72 & 0.52 & \\
\hline 4 & 0.41 & 0.53 & 0.74 & 0.57 & \\
\hline 5 & 0.52 & 0.59 & 0.64 & 0.55 & \\
\hline
\end{tabular}


The original thickness of the specimen $=5.7 \mathrm{~mm}$, The final thickness of the specimen $=4.786 \mathrm{~mm}$, Reduction ratio $=16 \%$, time $=180 \mathrm{sec}$.

Table 11: Illustrates the Dimensions of Specimen (S7) Before and After Non-Symmetrical Rolling for Gear Ratio Between Rollers (1.184).

\begin{tabular}{|c|c|c|c|c|c|}
\hline Square & $\begin{array}{c}\mathrm{dx} \\
(\mathrm{mm}) \\
\text { before } \\
\text { rolling }\end{array}$ & $\begin{array}{c}\mathrm{dx}(\mathrm{mm}) \\
\text { after } \\
\text { rolling }\end{array}$ & $\begin{array}{c}\text { dy } \\
\text { (mm) } \\
\text { before } \\
\text { rolling }\end{array}$ & $\begin{array}{c}\text { dy } \\
(\mathrm{mm}) \\
\text { after } \\
\text { rolling }\end{array}$ & $\begin{array}{c}\text { Square } \\
\text { row }\end{array}$ \\
\hline 1 & 0.49 & 0.70 & 0.55 & 0.43 & \multirow{6}{*}{ upper } \\
\hline 2 & 0.54 & 0.73 & 0.55 & 0.40 & \\
\hline 3 & 0.45 & 0.59 & 0.60 & 0.45 & \\
\hline 4 & 0.47 & 0.60 & 0.58 & 0.47 & \\
\hline 5 & 0.483 & 0.57 & 0.59 & 0.53 & \\
\hline 6 & 0.565 & 0.61 & 0.60 & 0.57 & \\
\hline 1 & 0.492 & 0.71 & 0.58 & 0.48 & \multirow{6}{*}{ Medium } \\
\hline 2 & 0.408 & 0.53 & 0.59 & 0.43 & \\
\hline 3 & 0.44 & 0.57 & 0.59 & 0.45 & \\
\hline 4 & 0.462 & 0.59 & 0.58 & 0.47 & \\
\hline 5 & 0.61 & 0.72 & 0.63 & 0.58 & \\
\hline 6 & 0.568 & 0.61 & 0.60 & 0.59 & \\
\hline 1 & 0.55 & 0.72 & 0.71 & 0.50 & \multirow{6}{*}{ Lower } \\
\hline 2 & 0.59 & 0.73 & 0.71 & 0.522 & \\
\hline 3 & 0.425 & 0.55 & 0.66 & 0.524 & \\
\hline 4 & 0.52 & 0.62 & 0.72 & 0.59 & \\
\hline 5 & 0.60 & 0.69 & 0.70 & 0.605 & \\
\hline 6 & 0.64 & 0.69 & 0.72 & 0.655 & \\
\hline
\end{tabular}

The original thickness of the specimen $=6.08 \mathrm{~mm}$, The final thickness of the specimen $=5 \mathrm{~mm}$, Reduction ratio $=17.7 \%$, time $=215 \mathrm{sec}$.

Table12:Illustrates the Dimensions of Specimen (S8) Before and After Non-Symmetrical Rolling for Gear Ratio Between Rollers (1.184).

\begin{tabular}{|c|c|c|c|c|c|}
\hline Square & $\begin{array}{c}\mathrm{dx} \\
(\mathrm{mm}) \\
\text { before } \\
\text { rolling }\end{array}$ & $\begin{array}{c}\mathrm{dx}(\mathrm{mm}) \\
\text { after } \\
\text { rolling }\end{array}$ & $\begin{array}{c}\text { dy } \\
\text { (mm) } \\
\text { before } \\
\text { rolling }\end{array}$ & $\begin{array}{c}\text { dy } \\
(\mathbf{m m}) \\
\text { after } \\
\text { rolling }\end{array}$ & $\begin{array}{c}\text { Square } \\
\text { row }\end{array}$ \\
\hline 1 & 0.47 & 0.60 & 0.60 & 0.52 & \multirow{5}{*}{ upper } \\
\hline 2 & 0.39 & 0.49 & 0.61 & 0.51 & \\
\hline 3 & 0.59 & 0.72 & 0.61 & 0.52 & \\
\hline 4 & 0.43 & 0.50 & 0.66 & 0.60 & \\
\hline 5 & 0.59 & 0.59 & 0.59 & 0.56 & \\
\hline 1 & 0.54 & 0.68 & 0.65 & 0.58 & \multirow{3}{*}{ Medium } \\
\hline 2 & 0.50 & 0.62 & 0.72 & 0.62 & \\
\hline 3 & 0.62 & 0.74 & 0.75 & 0.67 & \\
\hline
\end{tabular}

\begin{tabular}{|l|l|l|l|l|l|}
\hline 4 & 0.53 & 0.58 & 0.74 & 0.67 & \\
\hline 5 & 0.58 & 0.60 & 0.69 & 0.66 & \\
\hline 1 & 0.49 & 0.59 & 0.49 & 0.41 & \\
\hline 2 & 0.49 & 0.57 & 0.55 & 0.47 & \multirow{2}{*}{ Lower } \\
\hline 3 & 0.51 & 0.58 & 0.55 & 0.47 & \\
\hline 4 & 0.48 & 0.52 & 0.60 & 0.53 & \\
\hline 5 & 0.56 & 0.59 & 0.56 & 0.52 & \\
\hline
\end{tabular}

The original thickness of the specimen $=5.64 \mathrm{~mm}$, The final thickness of the specimen $=4.94 \mathrm{~mm}$, Reduction ratio $=12.4 \%$, time $=180 \mathrm{sec}$.

Calculation of Strain Rate: The dimensions were measured in the $x$ and $y$ direction of the printed grid on the thickness of each specimen before and after performing the symmetrical and non- symmetrical rolling, this is for determination of the deformation in the forming region and calculation of strain and strain rates in those region. Figure 9 illustrates a sketch for calculating the time taken for first and second square. Dividing the equivalent strain by the deformation time gives the strain rate at that point. This method enables the calculation of the strain rate of each square through the forming region. Consequently, this method describes the change of the strain rate with change of angle. The entry angle may be determined from the following relationship:

$$
\theta=\sin ^{-1}\left(\sqrt{\frac{\Delta h}{R}}\right)
$$

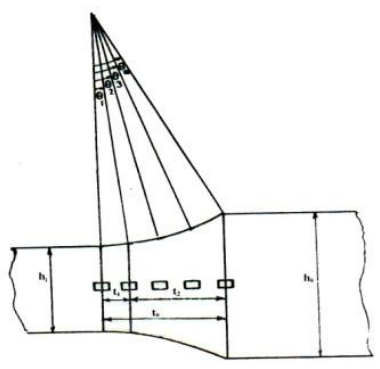

Fig 9 :Illustrates a sketch for calculating the time taken for first and second square.

Table 13 represents the calculation adopted to obtain the effective strain rate for specimen S1. This was applied on other specimen.

Table13:Illustrates the Calculation of the Effective Strain Rate for Specimen (S1) of Symmetrical Rolling Process.

\begin{tabular}{|c|c|l|c|c|c|c|}
\hline Square & $\begin{array}{c}\mathbf{d x}(\mathbf{m m}) \\
\text { after rolling }\end{array}$ & $\boldsymbol{\varepsilon}_{\text {effective }}$ & $\left(\boldsymbol{\varepsilon}_{\text {effective }}\right)_{\text {net }}$ & $\mathbf{t}_{\text {net }}(\mathbf{s e c})$ & $\dot{\boldsymbol{\varepsilon}}_{\text {effective }}(\mathbf{1} / \mathbf{s e c})$ & Square row \\
\hline 1 & 0.55 & 0.1777 & 0.0137 & 29.725 & 0.00046 \\
\hline 2 & 0.60 & 0.164 & 0.0298 & 30.45 & 0.00098 \\
\hline 3 & 0.66 & 0.1342 & 0.0255 & 30.015 & 0.00085 \\
\hline 4 & 0.65 & 0.1087 & 0.0517 & 30.45 & 0.0017 \\
\hline 5 & 0.56 & 0.057 & 0.057 & 28.42 & 0.0020 \\
\hline 1 & 0.46 & 0.1833 & 0.0124 & 25.23 & 0.00049 \\
\hline 2 & 0.51 & 0.1709 & 0.0371 & 29.58 & 0.00125 \\
\hline 3 & 0.65 & 0.1338 & 0.035 & 32.48 & 0.00108 \\
\hline 5 & 0.62 & 0.0988 & 0.0484 & 31.32 & 0.00154 \\
\hline
\end{tabular}

The original thickness of the specimen $=5.5 \mathrm{~mm}$, The final thickness of the specimen $=5 \mathrm{~mm}$, Reduction ratio $=9.1 \%$, entry angle $=$ 4.68 
Figure 10 illustrates the relationship between $\varepsilon_{z}$ with $\theta$ for the chosen specimen PS3in the experiment of plane strain. Figures 11 and 12 illustrates the relationship between the calculated strain rate through the deformations in the grid's dimension and between the angle of symmetrical

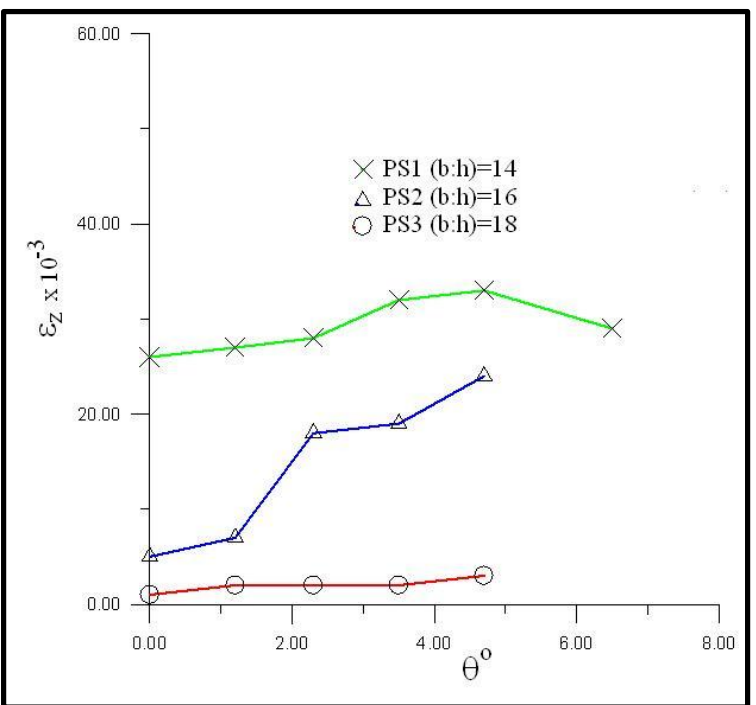

Fig 10: Illustrates the results of a deformation experiment by plane strain

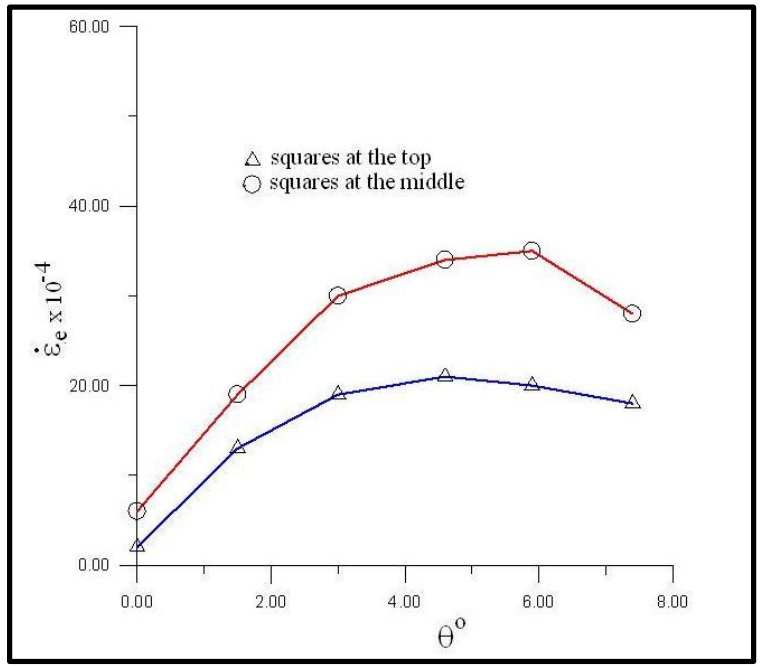

Fig 12:Illustrates the values of strain rate and their change with the roll angle for specimen S2 which is rolled at $21.3 \%$ thickness reduction ratio rolling for specimens S1 and S2. Figures 13 to 18 illustrates the relationship between the calculated strain rate at the deformation regions and the angles of non- symmetrical rolling for specimens S3 up to S8.

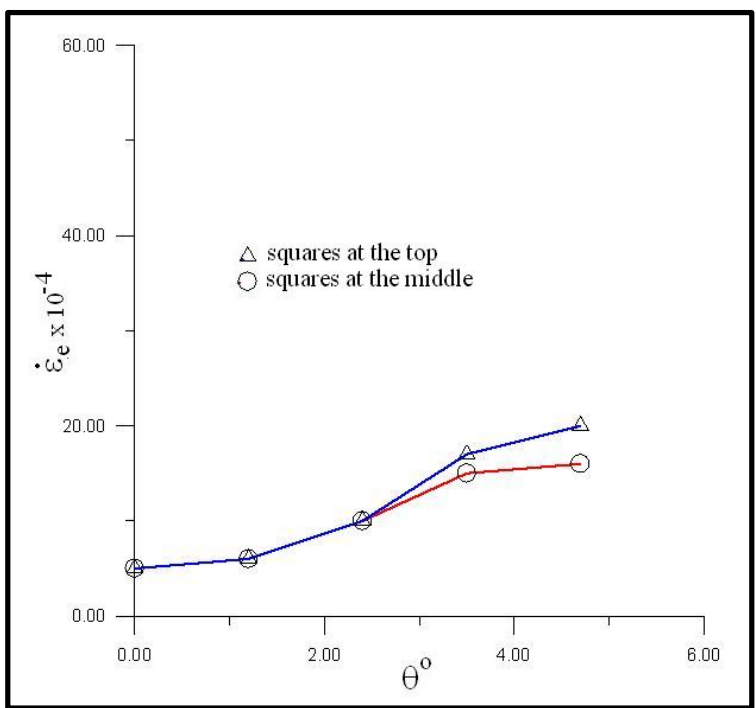

Fig 11: Illustrates the values of strain rate and their change with the roll angle for specimen $\mathrm{S} 1$ which is rolled at $9.1 \%$ thickness reduction ratio

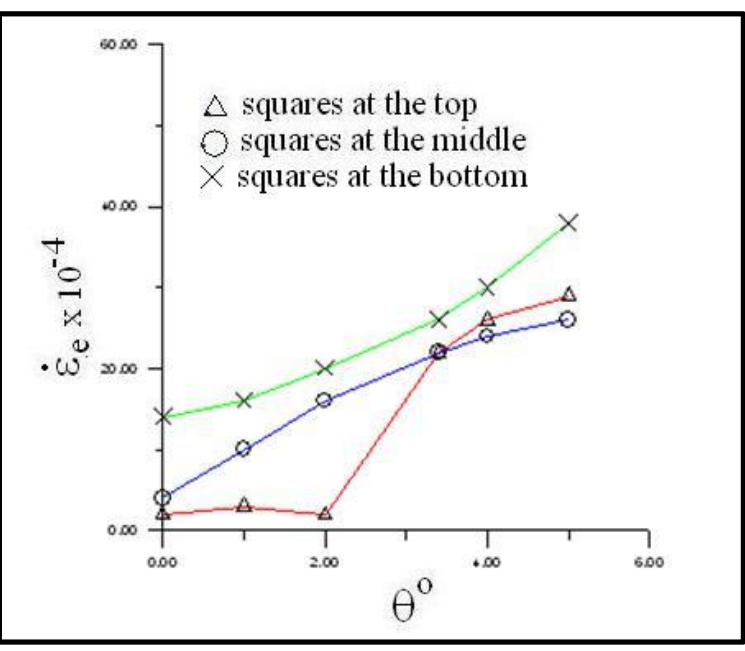

Fig 13: Illustrates the values of strain rate and their change with the roll angles for specimen S3 which is rolled at $10.3 \%$ thickness reduction rate and speed ratio between rolls of $\mathbf{1 . 0 7 5}$ 
Am. J. Sci. Ind. Res., 2013, 4(1): 59-74

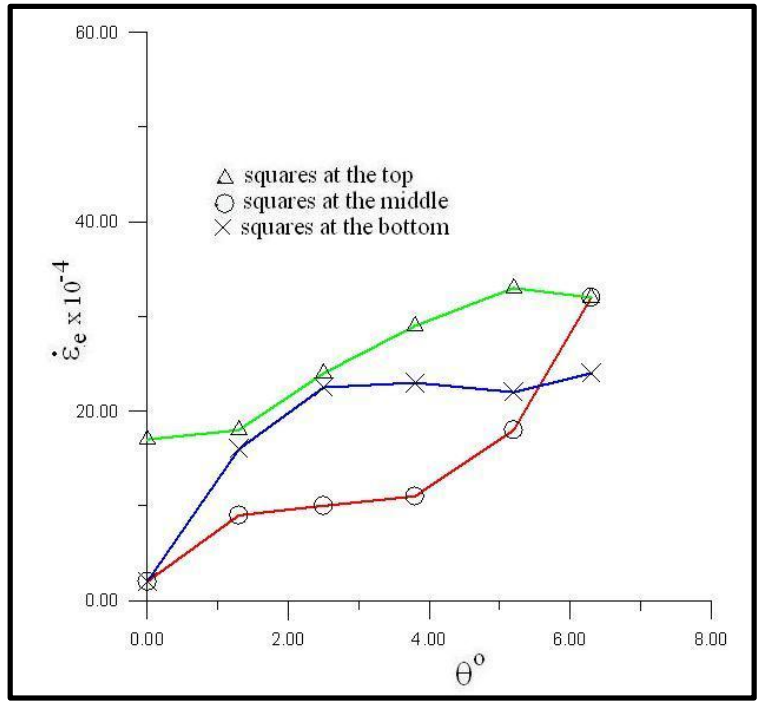

Fig 14:Illustrates the values of strain rate and their change with the roll angles for specimen S4 which is rolled at $16.3 \%$ thickness reduction rate and speed ratio between rolls of $\mathbf{1 . 0 7 5}$

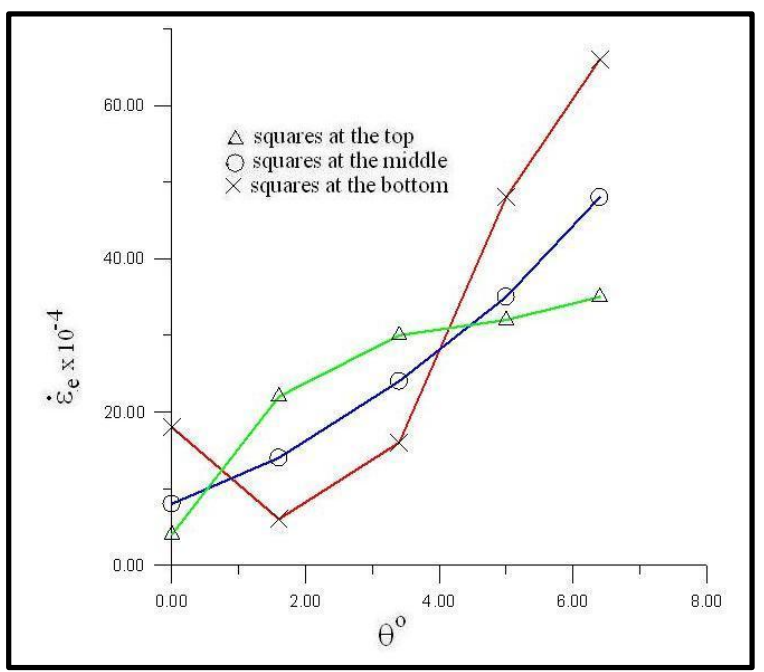

Fig 16:Illustrates the values of strain rate and their change with the roll angles for specimen S7 which is rolled at $17.7 \%$ thickness reduction rate and speed ratio between rolls of 1.184

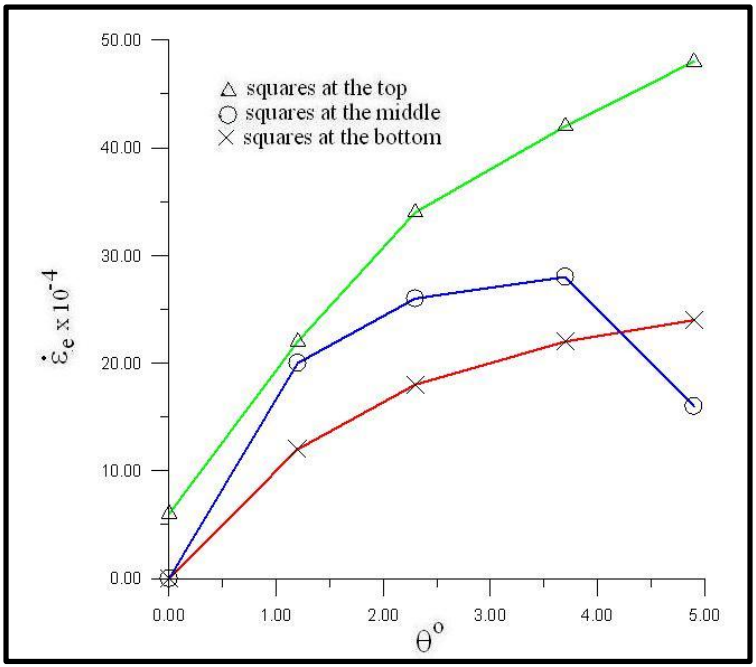

Fig 14:Illustrates the values of strain rate and their change with the roll angles for specimen S4 which is rolled at $16.3 \%$ thickness reduction rate and speed ratio between rolls of $\mathbf{1 . 0 7 5}$

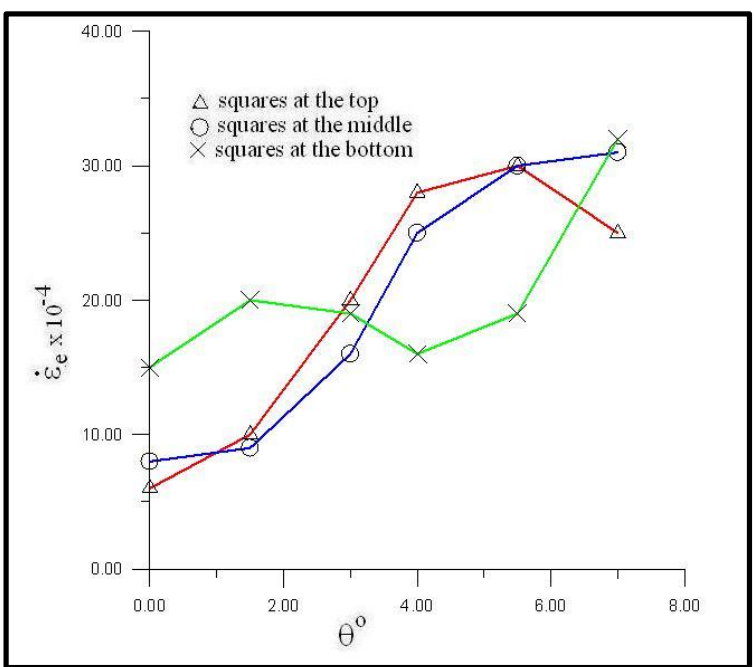

Fig 17:Illustrates the values of strain rate and their change with the roll angles for specimen S7 which is rolled at $\mathbf{1 7 . 7} \%$ thickness reduction rate and speed ratio between rolls of $\mathbf{1 . 1 8 4}$ 
Am. J. Sci. Ind. Res., 2013, 4(1): 59-74

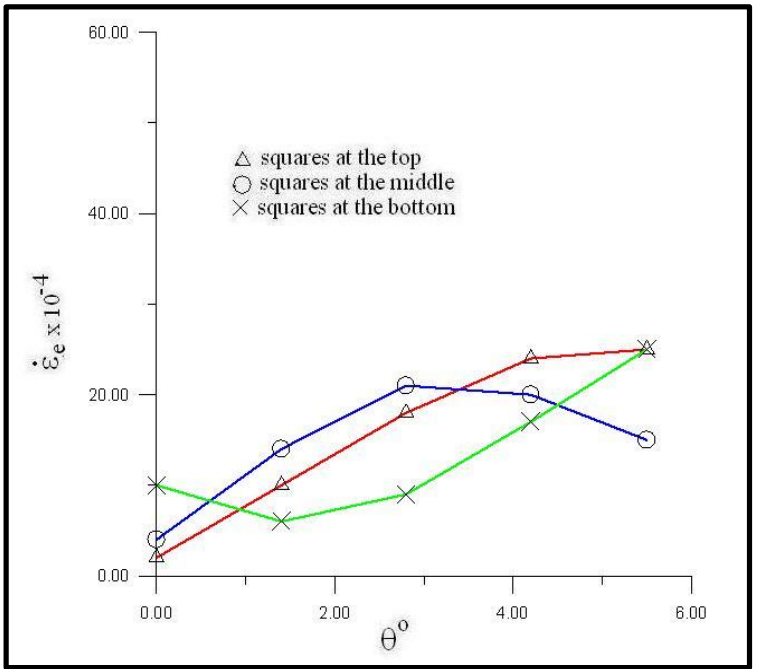

Fig 18: Illustrates the values of strain rate and their change with the roll angles for specimen S8 which is rolled at $\mathbf{1 2 . 4} \%$ thickness reduction rate and speed ratio between rolls of 1.184

\section{DISCUSSION}

It is well known that the metallic specimen is exposed to three directional (lateral, Iongitudinal, thickness- wise) deformation during rolling. This imposes studying the three directional strains. Calculations of the two types of rolling are complicated. In order to simplified such calculations, researchers often avoid studying the three directional rolling operation, their studies are restricted to two dimension only. This requires that strain in the third direction be equal to zero (i.e. plane strain). To determination of the dimensions of the used specimen and the ratio between the width $b_{0}$ to its thickness $h_{0}$ insures the case of deformation by plane strain. Thus, three specimens were chosen with different $b_{0} / h_{0}$ ratios. Rolling processes were performed on them with close thickness reduction ratios after printing a grid on its thickness. The three- directional strains $\left(\varepsilon_{z}, \varepsilon_{y}\right.$ and $\varepsilon_{\mathrm{x}}$ ) were calculated. The following was evidenced: ; the values of lateral strains for PS1 and PS2 specimens are of high value and do not meet the condition of plane strain; PS3 specimen (whose dimensions before and after rolling, were identified in Table 4; they are near zero). The dimension of the latter in which the ratio $b_{0} / h_{0}=18$ fold. Thus, these dimensions were adopted for determination of the dimension of the specimen under study,

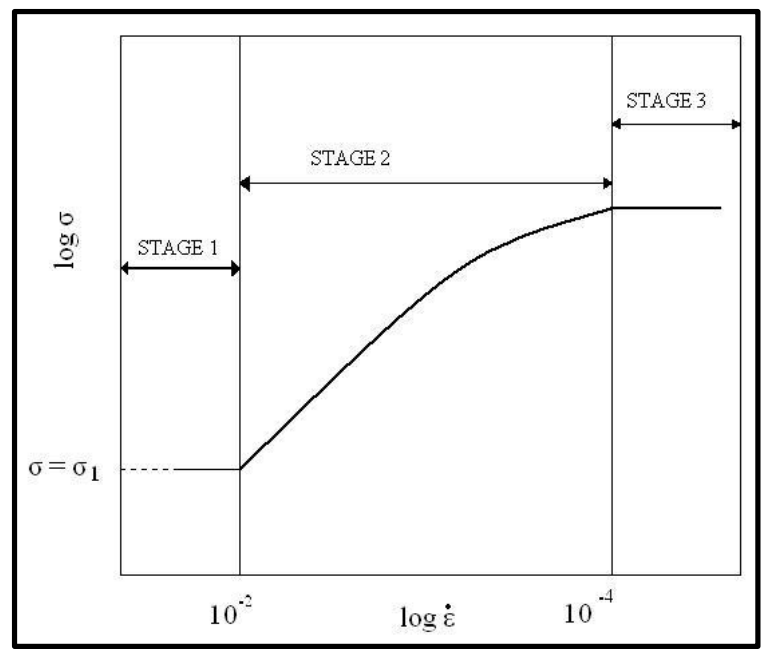

Fig 19: Illustrates the relationship between log stress verses log strain for superplastic materials.

because they meet the condition of plane strain. The aim of experiments of symmetrical and nonsymmetrical rolling is to find the strain rates generated at the different forming zones, method of describing the distribution of those rates along the deformation zones and compression thereof with the theoretical results in former researches. These results depend on the mathematical relationship that describes the distribution of strain rates throughout the deformation zone. For symmetrical rolling process, Alto, 1976, [4] adopted a definition for explication of strain rates. It depends on introduction of the angle in which the neutral plane $\left(\mathrm{Y}_{\mathrm{n}}\right)$ is located and the descriptive equation is:

$\dot{\varepsilon}_{e}=\frac{4}{\sqrt{3}} \frac{1+\tan ^{2} \gamma_{n}}{\sqrt{\frac{R}{h_{1}}+\tan ^{2} \gamma_{n}}} \frac{V_{p}}{h_{1}} \frac{\tan \gamma}{\left(1+\tan ^{2} \gamma_{n}\right)^{2}}$

where $(\mathrm{Y})$ is defined as $\tan \gamma=\sqrt{\frac{R}{h}} \tan \theta, \mathrm{V}_{\mathrm{p}}=$ linear velocity of the roll

The equation of the researcher Alto, geometrically describes the value of $\left(\dot{\varepsilon}_{e}\right)$ in the symmetrical rolling process for metals in general. It does not take into consideration the type of the rolled metal or alloy and the properties of the mechanical behavior. The latter is an indicator for the behavior of any metal or alloy during deformation. The metal used in this research is a lead-tin superplastic alloy. Its 
deformation is associated with the strain rates, thus, it is expected that the deformation in the rolling process for that material (and, consequently, the calculated strain rates for that deformation) would be different by a specific ratio. This is so because deformation in the specimen expresses the characteristics of the special mechanical behavior upon deformation. Anyway, that equation was represented by a curve of change of $\left(\dot{\varepsilon}_{e}\right)$ with the different angles of rolling. The aim is comparison with the equation of the researcher Alto [4] and with the experimentally calculated $\left(\dot{\varepsilon}_{e}\right)$ for the different thickness reductions ratios. Two symmetrical rolling experiments were performed with $9.5 \%$ and $21.3 \%$ thickness reduction ratios, the choice of these ratios was to have them chose to those implemented by the researcher Alto. Figures 11 and 12 illustrate the relationship of the calculated strain ratios in the different deformation zones verses their change with the rolling angles for both specimen. Each figure shows two curves; one curve represents the calculated in the horizontal row of squares near the top roll surface while the other curve represents the calculated in the horizontal row of squares near the bottom roll surface. The values of those strain rates in those rows were irregular. Therefore, the choice was for the average of such values and representation of its change with the different angles of rolling. The purpose thereof is to facilitate comparison with the curve obtained by the researcher Alto. Figures 19 and 20 illustrate the curve of the relationship between $\left(\dot{\varepsilon}_{e}\right)$ for the three above values and angles of symmetrical rolling. Figure 19, the curve of the average values of the calculated strain rates clearly approaches both theoretical curves. This confirm that the experimental experiments and measurements adopted in this experiment is up to an acceptable level of accuracy and that the deviation from the theoretical curve is due to deformation measurement accuracy in square at angle 2.4 (as illustrated in Figure 11). This is due to non-guarantee of fixation of location of measurement at the same point before and after rolling because of the deformation in the same square in the rolling process. The experimental results of the second experiment is illustrated in Figure 20. A reduction rate was used (with produced strain rates) higher than in the former experiment. It is observed from comparison of the theoretical values of both experiments that curve of second experiment is higher than that of the first experiment. This is due to the indicated reduction rates. Figures 19 and 20, confirm the experimental experiments, the curve of experimental value is identical with the theoretical value at the start of the specimen's entry at angle $7.3^{\circ}$. This continue until arriving angle $6^{\circ}$; then the curve departs with angle $1^{\circ}$ at which the curve starts to approach a little again until the exit zone. The high values of strain rates and deviation of the experimental curve from the theoretical curve is attributed to several effective factors, the most important factor is the ability to keep the specimen within the ambient temperature to guarantee no change in its crystallization properties due to re-crystallization which is large during the heating period. The experiment includes measurement of the squares' dimension by a direct light bulb microscope before the rolling process, the measurement takes a relatively long time for each specimen. This has an effect on exciting the process of re-crystallization of the specimen. This changes its mechanical properties and consequently, the strains occurring during rolling. For non-symmetrical rolling process, it is noted that the only difference in comparison of the results of the experimental experiments of nonsymmetrical rolling process from that symmetrical rolling process is that the comparison was between the average strain rates of the specimens and the theoretical curve adopted by the researcher Huda,1991, [12] as represented by the following equation:

$\dot{\varepsilon}_{e}=\frac{2}{\sqrt{3}}\left(V_{r 1}+V_{r 2}\right) \frac{\sin \theta}{h}$

The above equation is also a geometrical description for the deformation operation by rolling. The metal's speed at the start of rolling is less than that of the roll, this generates a frictional force between the surfaces of the specimen and the roll, this frictional force is towards the inside as if it is pulling the metal inwards. As the rolling process progresses, the specimen's speed increases until it reaches its maximum value at the exit. Thus, the direction of the frictional force change, outwardly after a point known the "neutral point" at which the speeds of the metal and the roll are equal. The experiments of non- symmetrical rolling processes are conducted in this research by different interrolls speeds. Therefore, the direction of the frictional forces between the specimens surface and that of each roll is different. Consequently, there is a zone at which the direction of the frictional forces at the two surfaces of the specimen change, this zone, called " cross section zone", is illustrated in Figure 10 and has an effect on the 
extent of the metal's deformation. The latter, consequently, has an effect on the extent of the specimen's curvature which increases with increase of the reduction ratio. Its direction is determined by thickness reduction, coefficient of friction, ratio of speeds between rolls [6]. The experiments of nonsymmetrical rolling processes in this research were conducted due to existence of curvature in the form of the specimen, the extent and direction of this curl differs from one specimen to another according to the working conditions of each specimen. A nonsymmetrical rolling process was performed on specimen S3 at 1.075 inter-rolls speed ratio and $10.3 \%$ thickness reduction ratio. Figure 21 illustrates the relationship between the average strain rates and rolling angle in the theoretical curve. This figure evidences that the curve of the average strain rates clearly approaches the theoretical curve; the little height in the curve of the average values is due to some high values in the squares of the bottom row. (as illustrated in Figure 18). This due to the curling of the form of specimen due to rolling; this curling is upwards, thus increasing the extent of deformation in the square close to the bottom roll relative to the other zones. Consequently, the average values of strain rates are increased. This is confirmed by the two researchers Johnson and Needham, 1982 with respect of dependence of curling direction on the reduction ratio in thickness and speed, friction between specimen's surface and the roll. By the same reduction ratio in speed and thickness (16.3\%), the specimen S4 was subjected to a nonsymmetrical rolling process. Figure 22 shows the theoretical curve and curve of the average values of strain rates and their change versus rolling angled. It is evident from the comparison between the theoretical and experimental curves the approach of curve of the average experimental values from the theoretical curve and its identical nature in the entry zone at angle $6.25^{\circ}$; this continue up to angle $4.75^{\circ}$ after which the curve start to depart a little up to the exist angle. This deviation between the two curves is due to the high values of strain rates in the square of the bottom row. This results from the downward direction of the curling in this case contrary to the direction of curling with case of the former specimen. This practically confirms that the increase of the reduction ratio upon constancy of speed reduction ratio between the two rolls leads to reversal of upward curling direction to downward direction, i.e. towards the fast roll. Specimen S5 was subjected for a rolling experiment of reduction ratio of $9.5 \%$ in thickness 1.13 in speed between the rolls. Comparison the calculated average value of strain rates with the theoretical curve clearly evidences the extent of remoteness in the curve of the average values from the theoretical curve, especially in the central zone. This is attributed to closeness of the top row from the roll surface and the little downward curling. The latter increases the values of deformation rates in the top surface as observed in Figure 15 with respect to the other zones. Such increase may be due to effect of both factors (curling and contact of row of squares with the roll surface counter to the direction of curling) in this specimen. The results of this experiment and the former one confirm that the curling of the specimen after exist from the roll gap depend on the reduction ratio in thickness and speed between the rolls. The curling's directions of specimens S3 and S5 are different in spite of the convergence of reduction ratio in thickness; this is due to increase reduction ratio of speed between the rolls for S5 relative to that for S3. S6 was subjected to nonsymmetrical rolling of $16 \%$ reduction ratio with the same reduction ratio in speed for the former specimen. Upon comparing the average values of strain rates for this specimen (as illustrated in Figure 24), it is evidenced relative increase in the average values from the theoretical value. This is attributed to the extent of upward curling in the form of the specimen due to the rolling process. Such a direction increased the value of strain rates in the bottom surface of the specimen. This is observed in comparison with the other zones in Figure 16. This increase is dependent on the extent of curling. Consequently the curve of the average values of strain rates is lifted. These are points outside of the curves of continuously fluctuating values due to non- guarantee of the constancy of the same zones of measurement zone before and after rolling. This is attributed to the non- uniformity of the geometrical patterns of the printing squares, this is associated with degree of accuracy in printing the grid. The pre- rolling deformed square experiences more deformation after rolling. This warrants the fluctuating values of strain rates of the different specimen. Results were obtained of the curling direction in the shape of the specimen and its variation with increased thickness reduction ratio. Thus, it is possible to control the linearity or the extent of curling of the workpiece by trying different thickness reduction ratios with constancy of speed reduction ratios, this is a useful industrial fact. In the following two experiments, a non- symmetrical 
rolling process for S7 and S8 specimens with reduction ratios of 1.184 for inter-rolls speed as well as $17.7 \%$ and $12.4 \%$ respectively for thickness of S7 and S8. Figures 17 and 18 illustrate the calculated strain rate in the different deformation zones for both specimens. Homogeneity in deformation zones of both specimens is evident, except existence of some outside points belonging to deformed squares, their total has on effect on description of the behavior of deformation described by the curves in both figures. A comparison is conducted between the theoretical curve specimen S7 and the average values of strain rates of Figure 25, there is an evident approach ( starting from the entry zone at angle

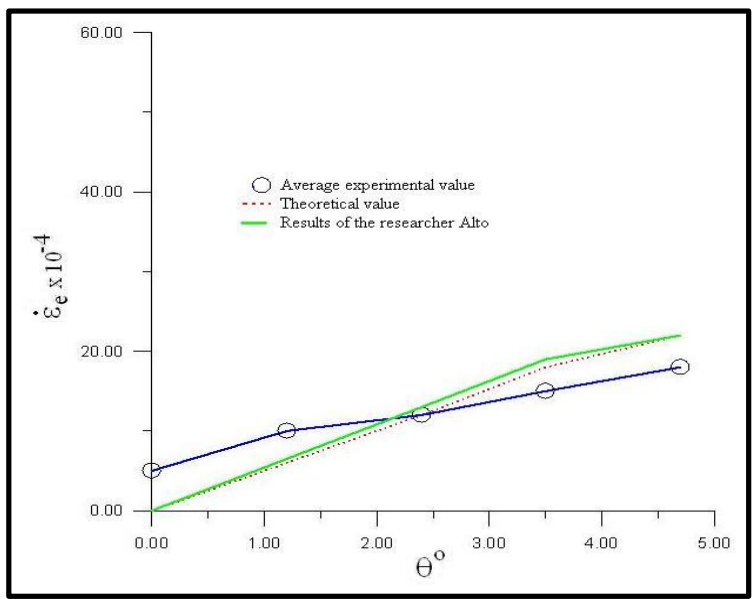

Fig 19: Illustrates relationship between the experimental values of strain rates and rolling angle as well as comparison thereof with the theoretical results of specimen $\mathrm{S} 1$

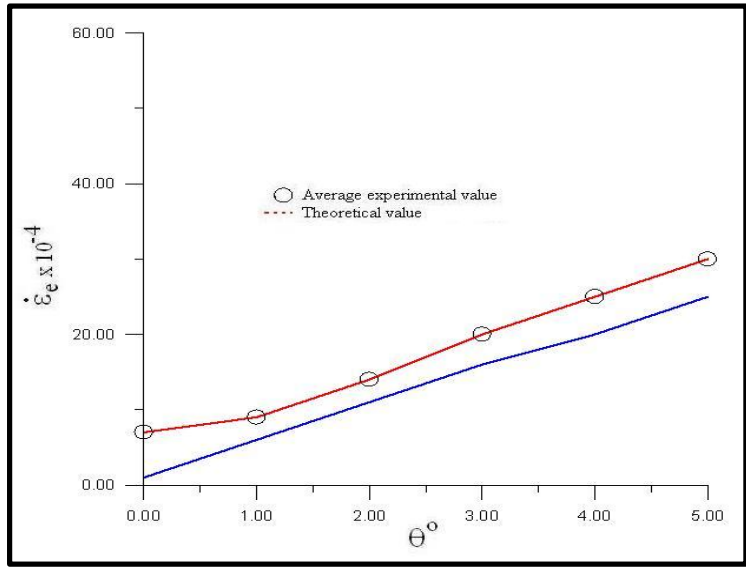

Fig 21:Illustrates relationship between the experimental values of strain rates and rolling angle as well as comparison thereof with the theoretical results of specimen $\mathrm{S3}$ $6.9^{\circ}$ up to angle $5.5^{\circ}$ ), which slightly departs until it keeps parallelism with the theoretical curve up to exist. A simple downward curling occurred in the shape of the specimen, due to the present high reduction ratio of $17.7 \%$. This had no great effect upon the values of the strain rates as is observed upon comparing of the curves of specimen S8 (illustrated by Figure 26, the curve of the average value of strain rates coincides with the theoretical curve at the entry zone at angle $5.5^{\circ}$, increasing up to angle $4^{\circ}$ and then slight deviation up to exit region. The increase of reduction ratios is thickness and inter-rolls speed in this experiments causes a very slight downward curling. This confirms again the curling direction is influenced by both factors.

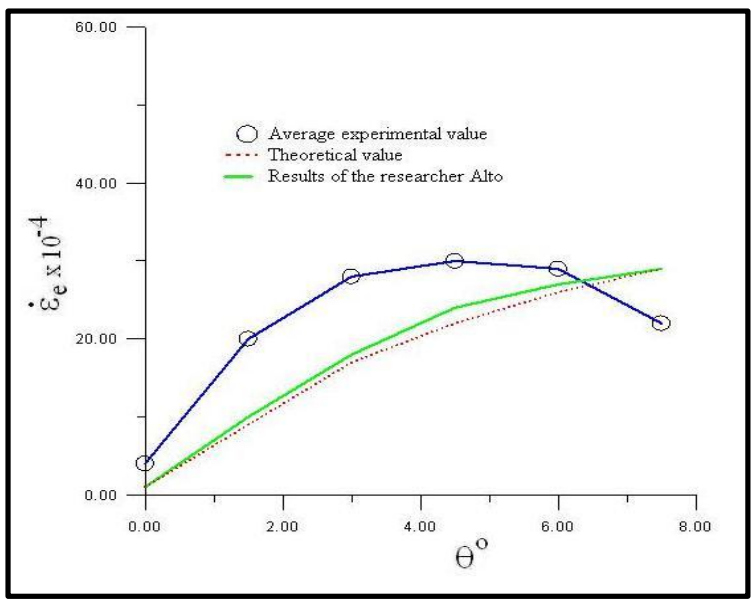

Fig 20: Illustrates relationship between the experimental values of strain rates and rolling angle as well as comparison thereof with the theoretical results of specimen $\mathbf{S 2}$

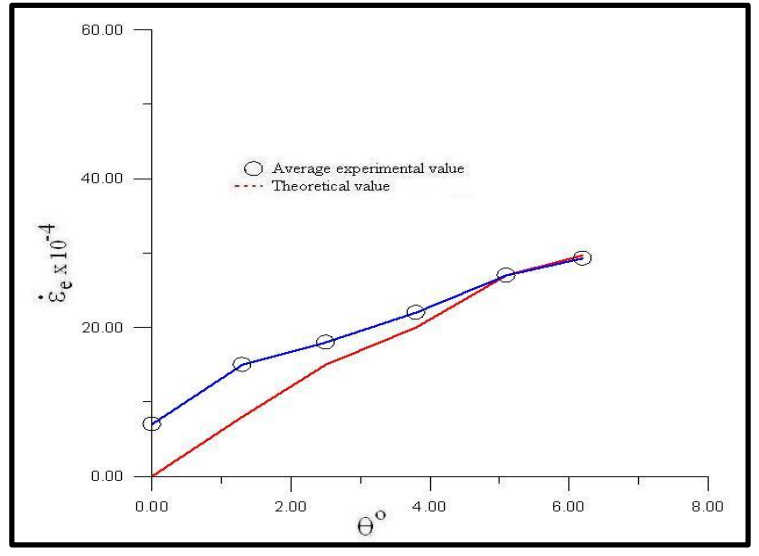

Fig 22: Illustrates relationship between the experimental values of strain rates and rolling angle as well as comparison thereof with the theoretical results of specimen S4 


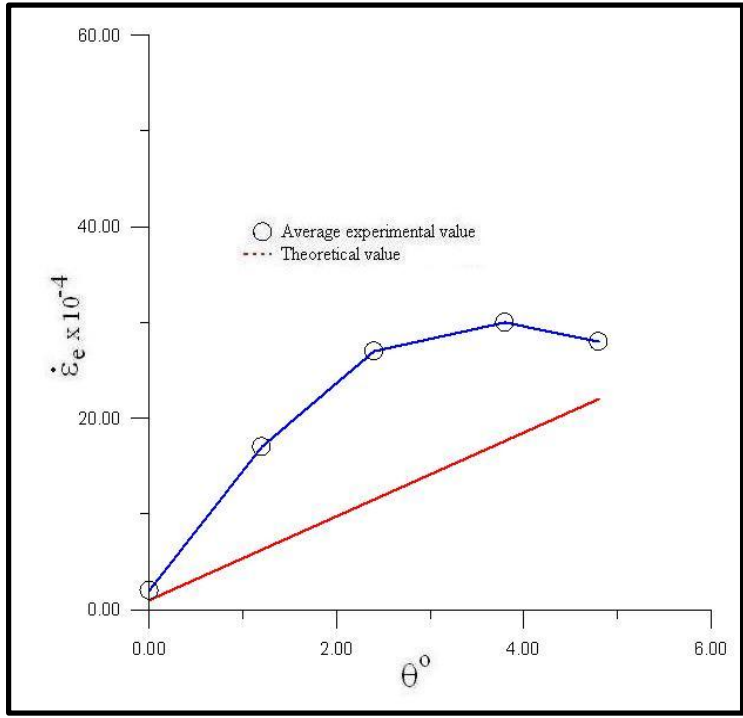

Fig 23: Illustrates relationship between the experimental values of strain rates and rolling angle as well as comparison thereof with the theoretical results of specimen S5

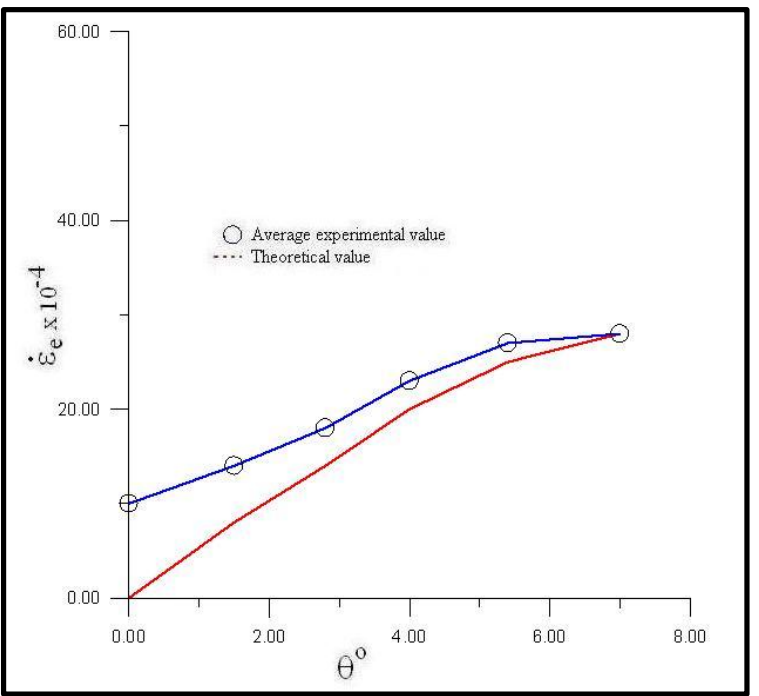

Fig 25: Illustrates relationship between the experimental values of strain rates and rolling angle as well as comparison thereof with the theoretical results of specimen $\mathrm{S7}$

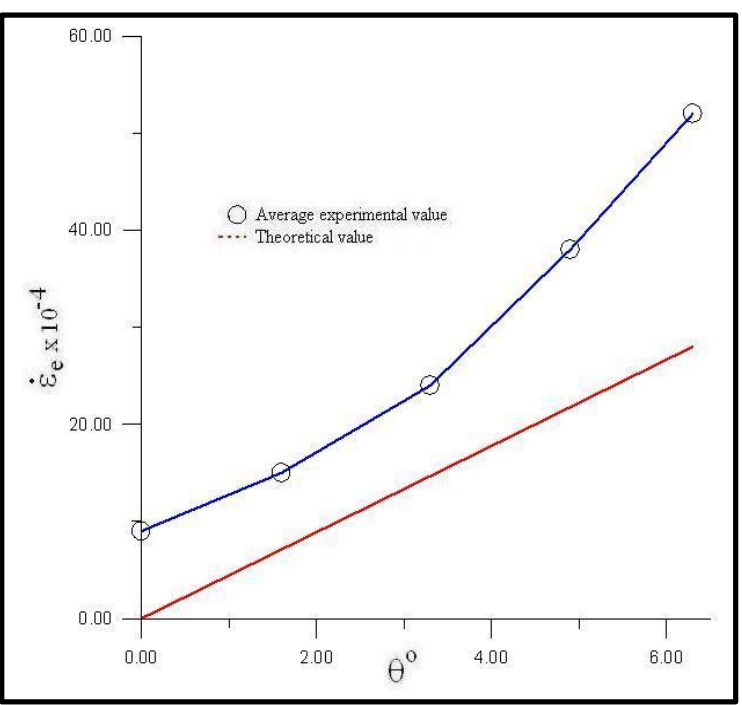

Fig 24: Illustrates relationship between the experimental values of strain rates and rolling angle as well as comparison thereof with the theoretical results of specimen $\mathrm{S} 6$

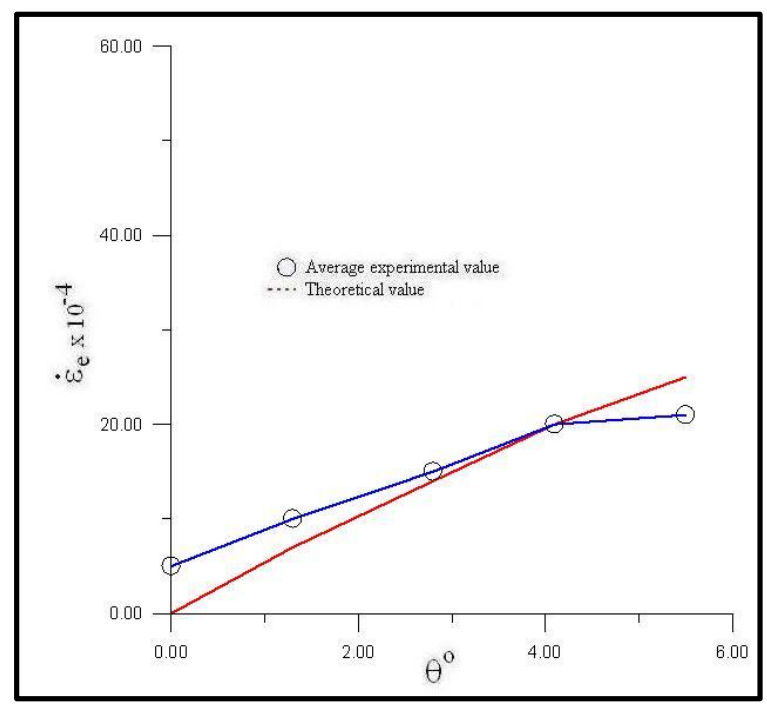

Fig 26: Illustrates relationship between the experimental values of strain rates and rolling angle as well as comparison thereof with the theoretical results of specimen $\mathbf{S 8}$ 


\section{CONCLUSIONS}

The following conclusions were attained from the discussion of the results:

1- The fabricated rolling machine achieved the required strain rates in the symmetrical and non- symmetrical rolling process for a superplastic lead-tin alloy of the range $\left(10^{-}\right.$ ${ }^{2}-10^{-4}$ ) $1 / \mathrm{sec}$ by operation at $0.0088 \mathrm{rpm}$ rotational speed of a $75 \mathrm{~mm}$ radius roll.

2- The experiments of symmetrical rolling by implementing a $9.1 \%$ reduction rate proved that the curve of average values of strain rates is higher than the theoretical curve by a percentage not great than $20 \%$ for a $21.3 \%$ thickness reduction rate, the percentage is greater.

3- The experiments of rolling process proved that the experimental curve represents the actual values that express the mechanical of the superplastic material. It is associated with the value of sensitivity coefficient for strain rate $(\mathrm{m})$ which increases as the value of the metal granules increases, the theoretical curves represents a geometric description that neglects the mechanical properties, that is why there is a slight difference in height between the two curves.

4- It was found experimentally that curvature in the shape of the non-symmetrically rolled specimen depend on thickness reduction ratio and inter-rolls speed, it increases with increase of the reduction ratio and its direction change with increase of inter- roll speeds. Thus, it is possible to obtain a linear shape for the specimen by choice of the suitable valuate for speed and thickness reduction ratios.

5- Acceptable results were obtained by measuring the dimension of a grid printed on the specimen's thickness before and after rolling. The purpose is to calculate the strain rate in the different deformation zones.

6- Symmetrical and non- symmetrical rolling experiments by $(21.3-9.1) \%$ reduction ratio and (1.184, 1.13, 1.075 and 1.0) inter- rolls speeds were performed. This proved that the greatest difference in values of strain rates was at angles $4.9^{\circ}$; it was $\left(38 \times 10^{-4}\right)$ $1 / \mathrm{sec}$. In comparison, the value of theoretical strain rate is $\left(25 \times 10^{-4}\right) 1 / \mathrm{sec}$.

\section{REFERENCES}

Burtsev K.,1973."Rolling Practice" , Mir Publisher , Moscow.

Orowan A. , 1943."The Calculation of Roll Pressure in Hot and Cold Flat Rolling", Proc. Instn. Mech. Engs.

Bland D.R. and Ford H., 1950." The Calculation of Roll Force and Torque in Cold Strip Rolling with Tensions" Proc. Instn. Mech. Engs.

Alto A., 1976. " An Approximate Analysis of the Strip Rolling of Rate Sensitive Materials Application to Superplastic Alloys" Int. Machine Tool Design Research, Vol. 16.

Dewhurst P., Collins I.F. and Johnson W., 1973."A Theoretical and Experimental Investigation in to asymmetrical hot rolling", Int. J. Mech. Sci., Vol. 16.

Johnson W. and Needham G., 1982. "Further Experiments in Asymmetrical Rolling", Int. J. Mech. Sci., Vol.8.

Chelyshev N.A. and LuzhnylA.P.,1988."Plastic Bending of Strip When Rolling in Rolls of Unequal Diameter", Steel in the USSR, Vol.1.

Nikolaev V.A. , 1978." Effect of Strip Inclination on Torque Distribution Between Rolls", Steel in the USSR, Vol. 9.

Nikolaev V.A., Volkov I.A. and EmchenkoV.I.,1988. "Effect of Vertical Deflection of Strip on Some Rolling Parameters", Steel in the USSR, Vol. 1 .

Pilling J. and Ridley N., 1989. "Superplasticity in Crystalline Solids", The Institute of Metals, London

Chung L. C., Cheng J. H, 2001. "Fracture criterion and forming pressure design for superplastic bulging", Journal of Material Science and Engineering, A333, 146-154.

Huda M., 1991. "Non- Symmetrical Rolling for Superplastic Material", M.Sc. thesis, University of Technology. 